\title{
Beam Test Results of Thin n-in-p 3D and Planar Pixel Sensors for the High Luminosity LHC Tracker Upgrade at CMS
}

\author{
I. Zoi ${ }^{* a}$, M. Boscardin ${ }^{b c}$, G. F. Dalla Betta ${ }^{d}$, M. Dinardo ${ }^{e}$, G. Giacomini ${ }^{b}$, D. Menasce ${ }^{f}$, \\ R. Mendicino ${ }^{d}$, M. Meschini ${ }^{g}$, A. Messineo ${ }^{h}$, L. Moroni ${ }^{f}$, S. Ronchin ${ }^{b c}$, D.M.S. \\ Sultan $^{d}$, L. Uplegger ${ }^{i}$, C. Vernieri ${ }^{i}$, L. Viliani ${ }^{g}$, D. Zuolo ${ }^{e}$ \\ a Universität Hamburg \\ E-mail: irene.zoi@cern.ch \\ b Fondazione Bruno Kessler, Trento, Italy \\ c TIFPA INFN, Italy \\ $d$ Università di Trento and TIFPA INFN, Italy \\ e Università di Milano Bicocca and INFN, Italy \\ $f$ INFN Milano Bicocca, Italy \\ g INFN, Firenze, Italy \\ $h$ Università di Pisa and INFN, Italy \\ i Fermi National Accelerator Laboratory, Batavia, IL, USA
}

This paper describes the development of new 3D and planar silicon pixel sensors designed for the Compact Muon Solenoid (CMS) Phase-2 Upgrade at High Luminosity LHC (HL-LHC). The project is funded by INFN and sensors are produced in collaboration with the FBK foundry. The HL-LHC will operate at an instantaneous luminosity approximately 5 times larger than the original LHC design, significantly increasing the number of concurrent collisions per bunch crossing, the integrated luminosity delivered to the experiments and, as a consequence, the radiation dose that the detectors will have to sustain. In order to cope with these future conditions, upgrades to the detectors are required. This is necessary for the pixel tracker that is the closest to the interaction point and will be replaced. In this paper, the results, from beam tests performed at Fermilab Test Beam Facility, of thin (100 $\mu \mathrm{m}$ and $130 \mu \mathrm{m}$ thick) $n$-in- $p$ type sensors, assembled into hybrid single chip modules bump bonded to the PSI46dig readout chip, will be presented. A comparison of the performances obtained with planar sensors before and after proton irradiation up to $3 \times 10^{15} \mathrm{n}_{e q} / \mathrm{cm}^{2}$ will be also discussed. The paper will also report the results obtained with the first 3D pixel sensors $130 \mu \mathrm{m}$ thick with columnar electrodes for different pixel cell prototypes. The novelty of the 3D prototypes is their small pixel cell size, ranging form the standard $100 \mu \mathrm{m} \times 150 \mu \mathrm{m}$, down to $50 \mu \mathrm{m} \times 50 \mu \mathrm{m}$ and $25 \mu \mathrm{m} \times 100 \mu \mathrm{m}$, which are the preferred dimensions in the high pile-up environment of the HL-LHC.

The European Physical Society Conference on High Energy Physics

5-12 July, 2017

Venice

*Speaker. 


\section{Introduction}

Major upgrades are planned for the Large Hadron Collider (LHC) at CERN allowing the accelerator to reach instantaneous peak luminosities of $5 \times 10^{34} \mathrm{~cm}^{-2} \mathrm{~s}^{-1}$ in the HL-LHC era [1]. In this regime an integrated luminosity of $300 \mathrm{fb}^{-1}$ per year will be delivered to the experiments and on average $\approx 140$ events per bunch crossing (pile-up) are foreseen, reaching eventually $\approx 200$ pile-up events in the highest luminosity scenario. To cope with these conditions experiments like ATLAS [2] and CMS [3] need to be upgraded. The inner tracker will be replaced with a suitably designed one. Pixel detectors in the innermost layers of the HL-LHC experiments are requested to maintain high tracking efficiency even at a fluence which can exceed $2 \times 10^{16} 1 \mathrm{MeV}$ neutron equivalent ${ }^{1} \mathrm{~cm}^{-2}$ (total estimated dose $\gtrsim 10 \mathrm{MGy}$ ). The tracker granularity has then to be increased, reducing the pixel area to $2500 \mu \mathrm{m}^{2}$. The new sensors are requested to have an active thickness between $100 \mu \mathrm{m}$ and $150 \mu \mathrm{m}$, possibly keeping the single pixel capacitance below $100 \mathrm{fF}$. The full sensor thickness should not exceed $200 \mu \mathrm{m}$ of silicon to be compatible with the low material budget allowance. Several R\&D projects dedicated to the development of the best suited sensors design are on going. The INFN R\&D described in this document is part of this effort. The pixel sensors designed in the INFN R\&D meet the requirements specified above, for both planar and 3D columnar devices. They have been produced at $\mathrm{FBK}^{2}$ foundry adopting the new single-side process DRIE technique (Deep Reactive Ion Etching) [4], on Silicon-Silicon Direct Wafer Bonded (SiSi DWB) material with active thickness $100 \mu \mathrm{m}$ and $130 \mu \mathrm{m}$. The sensors have been produced on 6" wafers in different sizes and pitches in order to be individually bump bonded to different ROCs (Read Out Chip). Designs are compatible with the present ROCs, FE-I4 for ATLAS [5] and PSI46dig [6] for CMS. In the following we will show results on planar and 3D single-chip module pixel sensors bump-bonded to the PSI46dig (100 $\mu \mathrm{m} \times 150 \mu \mathrm{m}$ pitch). Planar sensors were bump-bonded at Fraunhofer IZM (Berlin, Germany) while the Indium bump-bonding of 3D sensors was performed at Leonardo Company (Italy). They have been tested in 2015-17 at the Fermilab Test Beam Facility (FTBF) with a $120 \mathrm{GeV}$ proton beam. The facility, described in [7], houses a tracking telescope with a resolution of about $8 \mu \mathrm{m}$ on the Detector Under Test (DUT) which can be operated in a humidity and temperature controlled environment. All the devices have been characterised in terms of detection efficiency, which is computed as the ratio of the number of tracks reconstructed by the telescope having a corresponding hit on the detector, and the total number of tracks reconstructed by the telescope and traversing the detector, taking into account track pointing misplacements.

\section{Planar Pixel Sensors}

In this section, results from hybrid modules of the first FBK planar batch are presented. Sensors have been produced according to different layouts [8]. The measurements presented in the following are performed on a planar pixel sensor (wafer W30) with $100 \mu \mathrm{m}$ active thickness and $100 \mu \mathrm{m} \times 150 \mu \mathrm{m}$ pitch, chosen as representative example for the perfomance. It is characterised by a $50 \mu \mathrm{m}$ large gap between the $n^{+}$pixel implants surrounded by $\mathrm{C}$-shaped $p$-stop structures and no punch-through structure. To reduce discharges due to the high electric field between sensor and

\footnotetext{
${ }^{1} \mathrm{n}_{e q}$ in the following

${ }^{2}$ Fondazione Bruno Kessler, Trento (IT)
} 
ROC, a frame-shaped BCB (Benzo-Cyclo-Butene) layer has been deposited by IZM on both sensor and ROC periphery. The same device has been tested before and after irradiation at an average fluence of $3 \times 10^{15} \mathrm{n}_{e q} / \mathrm{cm}^{2}$, performed at IRRAD (CERN) in 2016 with $24 \mathrm{GeV} / \mathrm{c}$ protons.

Before irradiation the sensor was operated at a bias voltage of $40 \mathrm{~V}$ at room temperature; the detection efficiency, defined above, has been measured to be always above $99 \%$. The detection efficiency of the irradiated detector (cooled down to $-20^{\circ} \mathrm{C}$ ) has been measured at different bias voltages (Fig. 1a) and at different tilting angles with respect to the beam direction (bias voltage of $600 \mathrm{~V}$, Fig. 1b): it can sustain high voltages without charge multiplication and has a high efficiency, $97 \%$ at $600 \mathrm{~V}$.

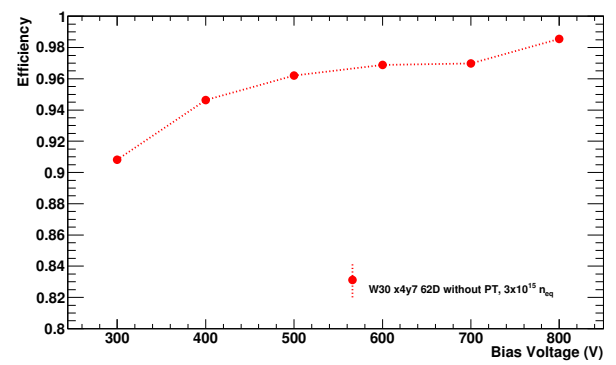

(a) Efficiency vs bias voltage

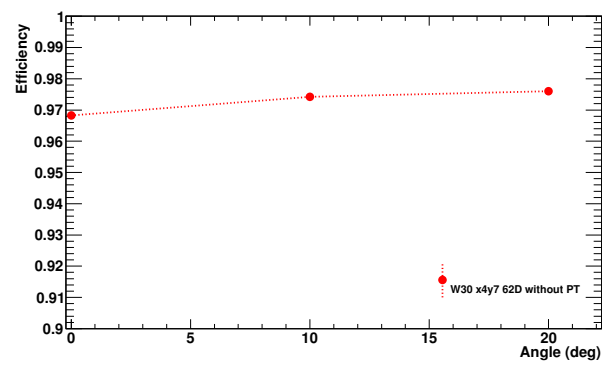

(b) Efficiency vs tilt angle

Figure 1: Results for a $100 \mu \mathrm{m}$ thick planar sensor. Overall detection efficiency at $3 \times 10^{15} \mathrm{n}_{e q} / \mathrm{cm}^{2}$ as a function of the bias voltage using orthogonal tracks (a) and as a function of the tilting angle at a bias voltage of $600 \mathrm{~V}$ (b) [9].

\section{3D Columnar Pixel Sensors}

The first INFN R\&D batch of 3D columnar pixel sensors was produced at FBK foundry in 2015. The 3D sensors have $p^{+}$(ohmic) columns passing through the high resistivity ( $\geq 3 \mathrm{k} \mathrm{Ohm} \cdot \mathrm{cm}$ ) active layer and reaching the low resistivity handle wafer, while the $n^{+}$(junction) columns are stopped at a distance of about $15-20 \mu \mathrm{m}$ from it, to avoid early breakdown [10]. The bias voltage is applied from the back-side through the handle wafer bulk, highly doped with Boron.

Devices with cells with one, two or three junction columns (1E, 2E, 3E) have been implemented, with the option to have the bump pad just on top of an ohmic column (BO) as an alternative to the standard off-column position (no BO). Sensors with pixel cell structures of $50 \mu \mathrm{m} \times 50 \mu \mathrm{m}$ or $25 \mu \mathrm{m} \times 100 \mu \mathrm{m}$, which have a dedicated adaptation metal network to allow a fraction of small pitch pixels to be readout by the PSI46dig ROC, have also been produced. In the small pitch case only one pixel out of six is read out, all the others being shorted together by the metal grid.

The results presented here refer to non irradiated sensors belonging to two non-thinned wafers (W76, W78) with $130 \mu \mathrm{m}$ active thickness ${ }^{3}$. The detection efficiency for orthogonal track has been measured to be above $99 \%$.

\footnotetext{
${ }^{3} 120 \mu \mathrm{m}$ effective active thickness due to Boron diffusion from the handle wafer.
} 


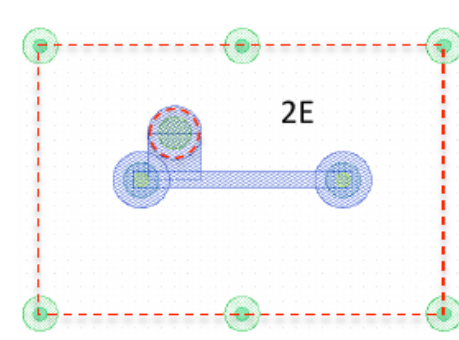

(a) Pixel cell design

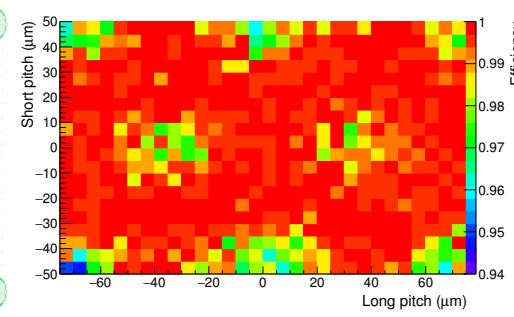

(b) 0 deg tilt angle

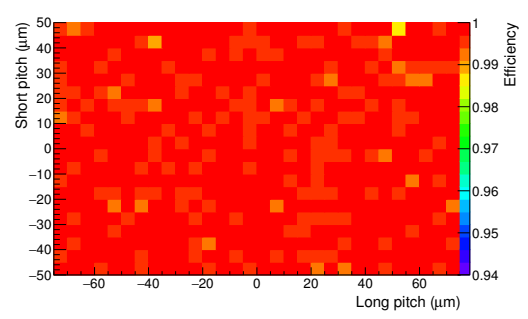

(c) 5 deg tilt angle

Figure 2: 3D pixel $100 \mu \mathrm{m} \times 150 \mu \mathrm{m}$ pitch 2E sensor, $130 \mu \mathrm{m}$ active thickness. Cell scheme with junction columns in blue, ohmic ones in green and bump pads circled in red (a). Cell detection efficiency for orthogonal (b) and 5 degrees inclined (c) tracks [11].

Detection cell efficiency as a function of the track impact position, averaged on all the cells, is shown in Fig. 2 for a 3D sensor with $100 \mu \mathrm{m} \times 150 \mu \mathrm{m}$ pitch and two electrodes (Fig. 2a). The column positions are still visible for orthogonal tracks (Fig. 2b) but for 5 degrees incident tracks, 3D pixels recover full efficiency (Fig. 2c).



(a) Planar

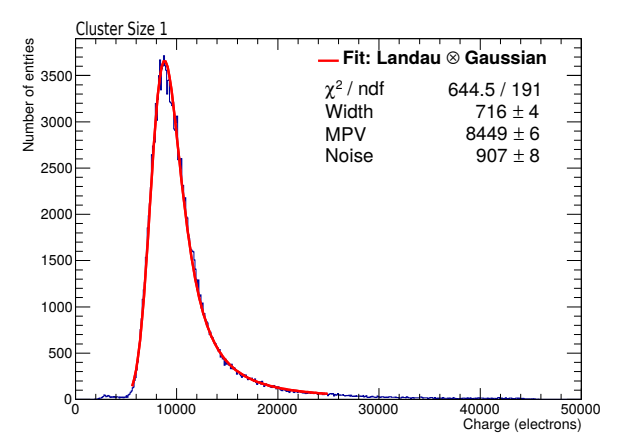

(b) $3 \mathrm{D}$

Figure 3: Collected charge distributions for a planar sensor operated at 100V (a) and a 3D sensor (b) operated at $40 \mathrm{~V}$, with same active thickness $(130 \mu \mathrm{m})$ [9].

Figure 3 shows the collected charge distributions for a planar sensor (Fig. 3a) and the above mentioned 3D sensor (Fig. 3b). They have been fitted with a Landau function convolved with a Gaussian, in red. The Most Probable Value ("MPV") of the Landau and the $\sigma$ of the Gaussian ("Noise") are given. The sensors have the same thickness $(130 \mu \mathrm{m})$ and collect the same charge but the planar sensor is operated at $100 \mathrm{~V}$ while to the $3 \mathrm{D}$ sensor a lower voltage of $40 \mathrm{~V}$ is applied.

Small pitch 3D pixel sensors have also been tested and representative examples (from wafer W76) are shown in Fig. 4. Top figures refer to sensors with $25 \mu \mathrm{m} \times 100 \mu \mathrm{m}$ pitch: Fig. 4a and Fig. $4 \mathrm{~b}$ are two examples of different pixel cell designs and options, as the bump can be either on top of the junction column (BO) or displaced from it; Fig. 4c shows the distribution of the charge collected by the sensor with $2 \mathrm{E}$ BO configuration (bias voltage of $40 \mathrm{~V}$ ), where the secondary peak at low charge values is due to the contribution of not read out adjacent pixels; the most probable value of the Landau distribution as a function of the bias voltage for three sensors with different 
layouts is in Fig. 4d. The three configurations differ for the number of charge collecting electrodes (1E or $2 \mathrm{E}$ ) and for the position of the bump. All three sensors are found to collect a comparable amount of charge within the systematic uncertainties, which are evaluated to be around 5\%, mainly due to the readout chip calibration procedures. Bottom figures are for a $50 \mu \mathrm{m} \times 50 \mu \mathrm{m}$ pitch sensor: Fig. 4e shows the pixel cell design, Fig. $4 \mathrm{f}$ the distribution of the charge collected by the sensor and Fig. 4g the most probable value of the Landau distribution as a function of the bias voltage. Variations in the amount of collected charge between sensors with different pixel sizes are due to the different impact of the not read out cells.

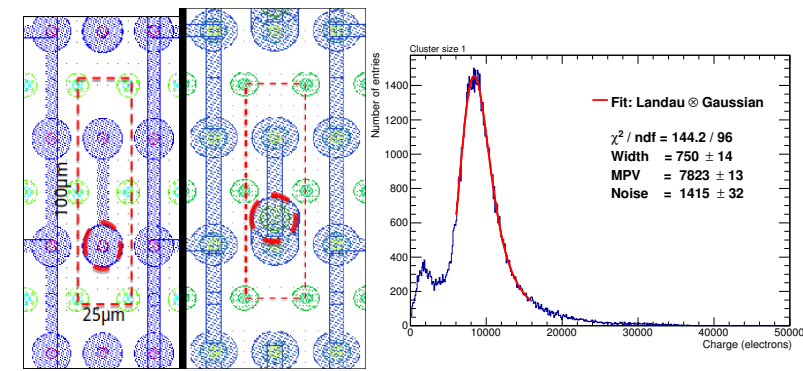

(a) $\mathrm{BO}$ (b) no $\mathrm{BO}$ (c) Collected charge distribution, $2 \mathrm{E} \mathrm{BO}$

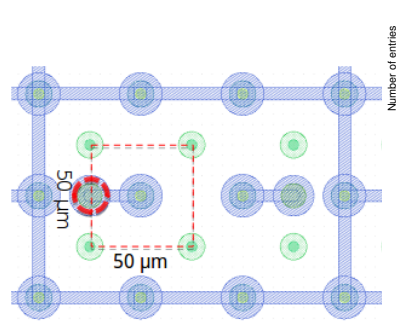

(e) Pixel cell design



(f) Collected charge distribution



(d) Collected charge at different bias

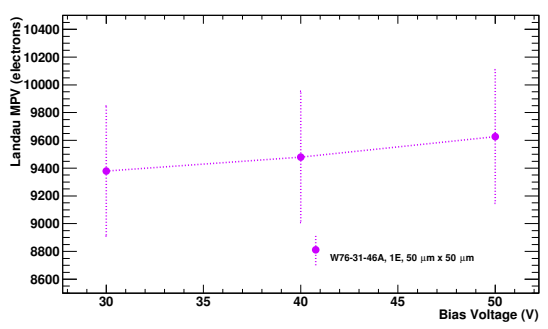

(g) Collected charge at different bias

Figure 4: Pixel cell designs and charge collection properties of different small pitch sensors (130 $\mu \mathrm{m}$ thickness), $25 \mu \mathrm{m} \times 100 \mu \mathrm{m}$ pitch at the top and $50 \mu \mathrm{m} \times 50 \mu \mathrm{m}$ pitch at the bottom [9].

\section{Conclusions}

The first results on planar and "single side process" 3D pixels produced in INFN-FBK R\&D show excellent sensor performance. The planar irradiated modules reach a detection efficiency of $97 \%$ at $600 \mathrm{~V}$ bias; irradiations of both planar and 3D pixels at higher fluences are on going. New 3D BO small pitch pixel sensors with the bump pad on the junction column have been produced and tested on beam for the first time and they are working according to our best expectations. The results obtained in a beam test before irradiation and the intrinsic radiation tolerance of 3D sensors confirm they are very good candidates for the innermost pixel detector layers at the HL-LHC. 


\section{References}

[1] G. Apollinari et al., High-Luminosity Large Hadron Collider (HL-LHC) Technical Design Report V. 0.1, CERN-2017-007-M, [doi 10.23731/CYRM-2017-004].

[2] Aad, G. et al. [ATLAS Collaboration], The ATLAS Experiment at the CERN Large Hadron Collider, JINST 3 (2008) S08003.

[3] R. Adolphi et al. [CMS Collaboration], The CMS experiment at the CERN LHC, JINST 3 (2008) S08004.

[4] G. F. Dalla Betta et al., Development of a new generation of 3D pixel sensors for HL-LHC, Nucl. Instrum. Meth. A824 (2016) 386-387, [doi $10.1016 / j . n i m a .2015 .08 .032$ ]

[5] The ATLAS IBL Collaboration, Prototype ATLAS IBL Modules using the FE-I4A Front-End Readout Chip, JINST 7 (2012) P11010.

[6] H.C. Kästli, Frontend electronics development for the CMS pixel detector upgrade, Nucl. Instrum. Meth. A731 (2013) 88.

[7] L. Uplegger et al., The pixel tracking telescope at the Fermilab Test Beam Facility, Nucl. Instrum. Meth. A811 (2016) 162-169, [doi $10.1016 /$ j.nima.2015.12.003].

[8] M. Meschini et al., The INFN-FBK pixel R\&D program for HL-LHC, Nucl. Instrum. Meth. A831 (2016) 116-121, [doi 10.1016/j.nima.2016.05.009].

[9] M. Meschini et al., Pixel Detector Developments for Tracker Upgrades of the High Luminosity LHC, CMS-CR-2017-190, https: / / cds. cern. ch/record/2276248

[10] G. F. Dalla Betta et al., The INFN-FBK "Phase-2" R\&D program, Nucl. Instrum. Meth. A824 (2016) 388-391, [doi 10.1016/j.nima.2015.08.074]

[11] CMS Collaboration, The Phase-2 Upgrade of the CMS Tracker - Technical Design Report, CERN-LHCC-2017-009, CMS-TDR-17-001 